\title{
Custo-efetividade do uso da tomografia de emissão de pósitrons na detecção de recorrência do câncer diferenciado de tireoide
}

I 1 Rosângela Caetano, ${ }^{2}$ Aline Navega Biz, ${ }^{3}$ Luciene Fontes Schluckebier,

${ }^{4}$ Rondineli Mendes da Silva, ${ }^{5}$ José Ueleres Braga, ${ }^{6}$ Cláudia Regina Garcia Bastos I

Resumo: Avaliou-se a custo-efetividade da adição da tomografia de emissão de pósitrons ( ${ }^{18} \mathrm{FDG}$-PET-TC) aos métodos convencionais na detecção de recorrência de cânceres diferenciados de tireoide. $\mathrm{O}$ modelo analítico de decisão representou coorte hipotética de pacientes adultos, de alto risco pela estratificação inicial, submetidos a tireoidectomia total e ablação com I ${ }^{131}$. A estratégia convencional de detecção foi comparada à adição da ${ }^{18}$ FDG-PET-TC aplicada aos indivíduos com resultados de cintigrafia com ${ }^{131}$ negativos. O horizonte temporal foi de dez anos. Utilizou-se a perspectiva do Sistema Único de Saúde (SUS) e, como medida de efetividade, os casos adicionais diagnosticados pela inclusão da PET-TC. Foram considerados apenas os custos médicos diretos. O custo do PET-TC foi estimado por microcustos; os demais custos foram obtidos das tabelas de pagamento de procedimentos do SUS. Custos e benefícios foram descontados em 5\%. Realizaram-se análises de sensibilidade determinística univariada e probabilística. Detectaram-se 1.875 casos de recorrência com a estratégia convencional. Uso da ${ }^{18}$ FDG-PET-TC permitiu a detecção adicional de 13 casos, com custo de R\$477.633,05/caso detectado. Os parâmetros de maior impacto na análise foram: medidas de acurácia dos métodos convencionais, custo do PET-TC e taxa de desconto. Os custos da adição da PET-TC mostram-se significativos e sua introdução não é custo-efetiva.

Palavras-chave: câncer de tireoide; tomografia de emissão de pósitrons; ${ }^{18}$ FDG-PET-TC; custo-efetividade; avaliação de tecnologias biomédicas.

\author{
1 Instituto de Medicina Social, \\ Universidade do Estado do Rio \\ de Janeiro. Rio de Janeiro-RJ, \\ Brasil (caetano.r@globo.com) \\ ${ }^{2}$ Instituto de Medicina Social, \\ Universidade do Estado do Rio \\ de Janeiro. Rio de Janeiro-RJ, \\ Brasil (alinebiz@gmail.com). \\ ${ }^{3}$ Instituto Nacional do Câncer. \\ Rio de Janeiro-RJ, Brasil \\ (lucfontes@gmail.com). \\ ${ }^{4}$ Instituto de Medicina Social, \\ Universidade do Estado do Rio \\ de Janeiro; Escola Nacional de \\ Saúde Pública, Fiocruz. Rio de \\ Janeiro-RJ, Brasil (rondineli. \\ mendes@gmail.com).

\footnotetext{
${ }^{5}$ Instituto de Medicina Social, Universidade do Estado do Rio de Janeiro; Escola Nacional de Saúde Pública, Fiocruz. Rio de Janeiro-RJ, Brasil (ueleres@ gmail.com).

${ }^{6}$ Instituto de Medicina Social, Universidade do Estado do Rio de Janeiro; Conselho Regional de Farmácia. Rio de Janeiro-RJ, Brasil (crgbastos@gmail.com).
}

Recebido em: 13/06/2014 Aprovado em: 28/12/2015 


\section{Introdução}

O ritmo de expansão das despesas do setor de saúde não tem sido acompanhado pelo crescimento das economias, constituindo um dos principais desafios das políticas públicas atuais para garantir a equidade no acesso aos serviços e às novas tecnologias em saúde e, ao mesmo tempo, o uso racional dos recursos. Estudos de avaliação econômica, que examinam as consequências clínicas e custos das novas tecnologias, são ferramentas que podem auxiliar os gestores de saúde nas decisões relativas a sua incorporação nos sistemas de saúde, na medida em que permitem o exame da eficiência dos novos procedimentos vis-à-vis os já existentes (VIANNA; CAETANO, 2005).

A tomografia de emissão de pósitrons (PET scan) é uma tecnologia de alto custo e de recente introdução no país. Seu uso tem sido proposto em diversas aplicações oncológicas. Foi incluída na tabela de procedimentos do Sistema Único de Saúde (SUS) em abril de 2014, para as seguintes indicações: estadiamento clínico do câncer de pulmão de células não pequenas potencialmente ressecável; detecção de metástases hepáticas de câncer colorretal potencialmente ressecáveis; e estadiamento e avaliação da resposta ao tratamento dos linfomas Hodgkin e não Hodgkin (BRASIL, 2014a; 2014b; 2014c). Um dos gargalos para sua expansão nos serviços de saúde brasileiros - a oferta do ${ }^{18} \mathrm{~F}-\mathrm{FDG}$, radiofármaco utilizado nos usos oncológicos da PET - encontra-se superado com a queda do monopólio da União na produção de radiofármacos em 2006 (BRASIL, 2006). A maior oferta do insumo e a incorporação do procedimento aos sistemas de saúde público e privado têm estimulado a expansão da compra e instalação de equipamentos de PET e PET-TC no país e vêm produzindo pressóes para a ampliação das indicações da tecnologia.

Cânceres de tireoide constituem a malignidade endócrina mais comum (TUTTLE et al., 2010) e sua incidência vem aumentando em alguns países (ROSENBAUM; McHENRY, 2009). No Brasil, as estimativas de incidência do câncer para biênio 2016/2017 lançadas em novembro de 2015 preveem a ocorrência de 6960 novos casos de câncer tireoidiano em 2016, correspondendo a 1,2\% de todas os tumores não melanoma e representando a oitava neoplasia maligna mais frequente na população feminina (BRASIL/MS, 2015).

Os cânceres diferenciados de tireoide (CDT), constituídos pelos carcinomas papilares e foliculares, correspondem à maioria dos tumores e figuram entre 
as neoplasias malignas com maior índice de cura; aproximadamente $85 \%$ dos

pacientes são curados com cirurgia, ablação com iodo radioativo $\mathrm{I}^{131}$ e supressão do TSH (ATA et al., 2009).

Embora usualmente possuam bom prognóstico, recidivas são bastante frequentes. Cerca de $20 \%$ dos pacientes apresentarão recorrência local, no próprio leito tireoidiano ou em gânglios cervicais e de mediastino; e 5 a 10\% desenvolverão metástases à distância, mais comumente para pulmões, ossos e cérebro (SHOUP et al., 2003). Acompanhamento adequado pós-tratamento inicial do CDT para detectar doença persistente ou recorrente e metástases distantes é necessário, permitindo a tomada de decisões que possam modificar o curso da doença (PACINI et al., 2012).

É recomendado que o seguimento dos pacientes seja individualizado de acordo com o risco de recorrência da doença. Tradicionalmente, dosagens seriadas de tireoglobulina sérica (Tg), ultrassonografia cervical (US) e cintigrafia diagnóstica com ${ }^{131} \mathrm{I}$ ou ${ }^{123} \mathrm{I}$ (PCI) têm papel importante no seguimento pós-cirúrgico (LIND; KOHLFURST, 2006; ATA et al., 2009; PACINI et al., 2012).

Embora a PCI tenha ocupado, durante muitos anos, papel central no seguimento do CDT, suas indicações hoje são mais restritas. Ela permite a localização da recidiva local ou de metástases à distância e auxilia nas decisões subsequentes sobre o tratamento com iodo radioativo, mas seus resultados dependem da habilidade do tecido neoplásico em captar $\mathrm{I}^{131}$ na presença de concentrações elevadas de TSH e, também, do tamanho das metástases. Detecta recorrência de CDT com alta especificidade, mas apenas moderada sensibilidade (LIN; KOHLFURST, 2006) e pode mostrar resultados negativos em 10 a 20\% dos pacientes com níveis séricos detectáveis de Tg (MA et al., 2005).

PET scan utilizando ${ }^{18} \mathrm{~F}$-FDG teve as primeiras utilizações no CDT metastático há mais de 20 anos (JOENSUU; AHONEN, 1987), quando foi identificado que algumas lesões metastáticas eram visualizadas apenas com ${ }^{18} \mathrm{~F}-\mathrm{FDG}$, outras só com $\mathrm{I}^{131}$ e outras com ambos. Feine e colaboradores (1996) cunharam o termo "flip flop" para esse padrão de alternância de captação pelas metástases, onde a captação de FDG nas lesões iodo-negativas poderia representar crescimento de células tumorais mais agressivas (LIND et al., 2003).

A introdução dos sistemas híbridos de PET-TC a partir de 2001, que conjugam imagem anatômica e funcional e possuem melhor performance diagnóstica, 
permite localizar mais acuradamente lesōes e potencial infiltração para estruturas adjacentes, e facilita a interpretação das imagens em locais de captação fisiológica do FDG (NAHAS et al., 2005; RIEMANN et al., 2013).

A literatura tem apontado que inclusão da ${ }^{18}$ FDG-PET-TC no algoritmo diagnóstico pode conduzir a mudanças subsequentes no manuseio em 9 a 54\% dos casos (NAHAS et al., 2005; SIBILLE et al., 2010). Entretanto, nos diversos protocolos clínicos seu uso é proposto em pacientes de alto risco de recorrência, sobretudo quando as investigações usuais se mostram negativas (ATA et al., 2009; MILAS et al., 2011; LANG; LAW, 2011; PACINI et al., 2012). O protocolo clínico para carcinoma diferenciado da tireoide publicado pelo Ministério da Saúde brasileiro em janeiro de 2014 não faz menção ao uso desta tecnologia (BRASIL, 2014b).

Pesquisa da literatura nacional e internacional não permitiu identificar qualquer estudo de avaliação econômica sobre o uso da PET no CDT. Assim, este estudo teve por objetivo estimar a custo-efetividade do uso da ${ }^{18} \mathrm{FDG}$-PET adicionado aos métodos convencionais de detecção de recorrência do carcinoma diferenciado da tireoide, na perspectiva do SUS.

\section{Método}

Foi desenvolvido um modelo de decisão analítico, utilizando-se os softwares TreeAge Pro Suite ${ }^{\circledR}$ e Excel ${ }^{\circledR}$. A população de referência correspondeu a uma coorte hipotética de 10.000 pacientes adultos, de ambos os sexos, classificados como de alto risco de recorrência pela estratificação inicial, ${ }^{1}$ submetidos à tireoidectomia total seguida de ablação com iodo radioativo, que iniciam seguimento com vistas à detecção de possível recorrência metastática.

A estratégia convencional de detecção de recorrência do CDT (dosagem da tireoglobulina sérica, ultrassonografia cervical e pesquisa de corpo inteiro) foi comparada ao estadiamento convencional adicionado com a PET-TC, aplicada aos indivíduos com resultados de cintigrafia com $\mathrm{I}^{131}$ negativos.

O desfecho de interesse foi a detecção de casos adicionais de recorrência no CDT, considerando a potencialidade da ${ }^{18} \mathrm{FDG}$-PET em detectar metástases locorregionais e à distância previamente não suspeitadas pelas técnicas convencionais. O horizonte temporal da avaliação foi de dez anos, dividido em ciclos anuais, porque este corresponde à faixa onde se concentram a grande 
maioria das recorrências do CDT (HAY et al., 2002) e ao seguimento mínimo recomendado nas diretrizes clínicas brasileiras deste câncer (BRASIL, 2014b). As condutas clínicas e a sequência de testes diagnósticos consideram o disposto nos protocolos clínicos das Associações Europeia de Tireoide (PACINI et al., 2012) e Americana de Tireoide (ATA, 2009), e no documento brasileiro (BRASIL, 2014e).

Em ambos os braços, os pacientes iniciavam o seguimento pela realização de dosagem de Tg suprimida (isto é, em uso de reposição de hormônio tireoidiano) e US cervical, realizado nove meses após o tratamento com tireoidectomia total e ablação. Casos com resultados de Tg suprimida indetectável ou inferior a 1ng/ $\mathrm{ml}$ eram submetidos à suspensão do hormônio exógeno e repetiam a dosagem quando o TSH atingia níveis superiores a 30 UI (dosagem de Tg estimulada). Considerou-se que todos os pacientes eram testados para a presença de anticorpos anti-Tg, em cada dosagem de Tg sérica suprimida ou estimulada, e mostravam resultado negativo. Pacientes com Tg suprimida ou estimulada que possuíam US mostrando alterações estruturais sugestivas de recorrência em região cervical eram submetidos à punção aspirativa com agulha fina e citologia e mensuração da Tg no lavado de agulha (Tg-PAAF). Se a recorrência não era confirmada pelo Tg-PAAF, o paciente era monitorado dentro de um ano, com repetição das dosagens laboratoriais e do exame de imagem. Se a US não mostrava evidências de recorrência em região cervical e a $\mathrm{Tg}$ se mantinha elevada, o paciente era submetido à PCI diagnóstica. Casos detectados dessa maneira eram posteriormente submetidos ao tratamento indicado em função da localização da recorrência.

Os braços diferiam na situação dos resultados do exame cintigráfico com I ${ }^{131}$ se mostrarem negativos. No braço da detecção convencional (figura 1), estes casos podiam ser tratados empiricamente com iodoterapia ou permanecer em seguimento e realizar nova sequência de testes no espaço de um ciclo do modelo. No braço que incluía PET-TC (figura 2), apenas pacientes com resultados negativos à PCI realizavam este procedimento. Casos positivos à PET-TC foram considerados casos detectados, se o status da doença definido ao início da árvore fosse correspondente à recorrência. Exames cito ou histológico de confirmação dos achados PET não foram incluídos nas árvores de decisão. Casos que se mostravam negativos à PET (verdadeiro-negativos ou falso-negativos) podiam 
ser submetidos empiricamente à iodoterapia (nos casos verdadeiro-negativos, desnecessariamente) ou a monitoramento.

Para fins de simplificação do modelo, foi feito o pressuposto que nenhum paciente da coorte sairia do modelo por óbito, fosse pela doença específica ou por outras causas, dada a dificuldade de obter dados de mortalidade ajustados por situação de recorrência (grande variabilidade das taxas entre estudos e localização de recorrência do CDT).

Figura 1. Modelo de Decisão empregado no estudo de custo-efetividade. Estratégia convencional de detecção de recorrência do câncer diferenciado de tireoide

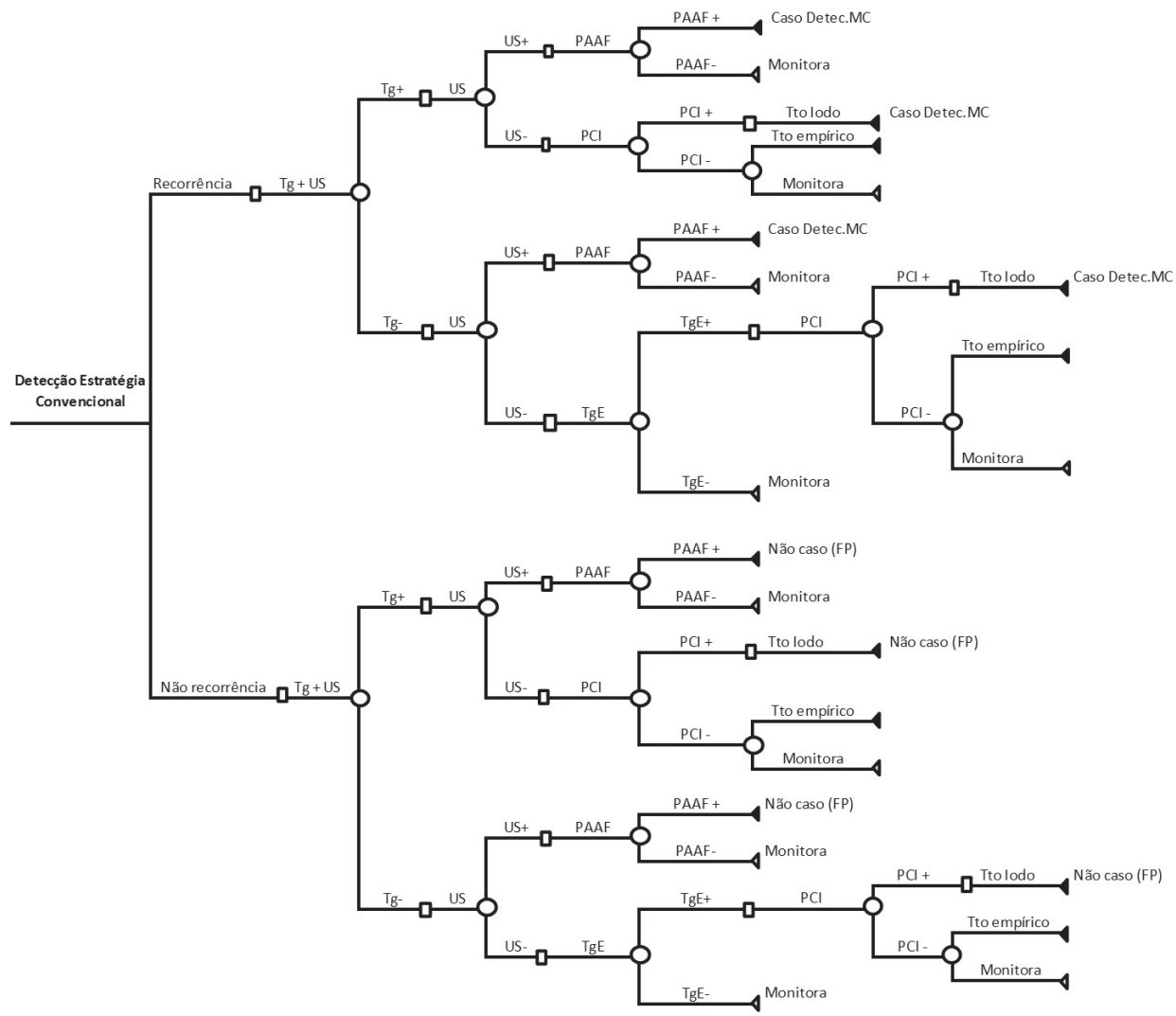

Legenda: FP - Falso-positivo; MC - Métodos Convencionais; PAAF - Punção aspirativa com agulha fina; PCI - Pesquisa de corpo inteiro; Tg - Tireoglobulina sérica suprimida; TgE - Tireoglobulina sérica estimulada; Tto - Tratamento com I ${ }^{131}$; US - Ultrassonografia. Fonte: elaboração própria. 


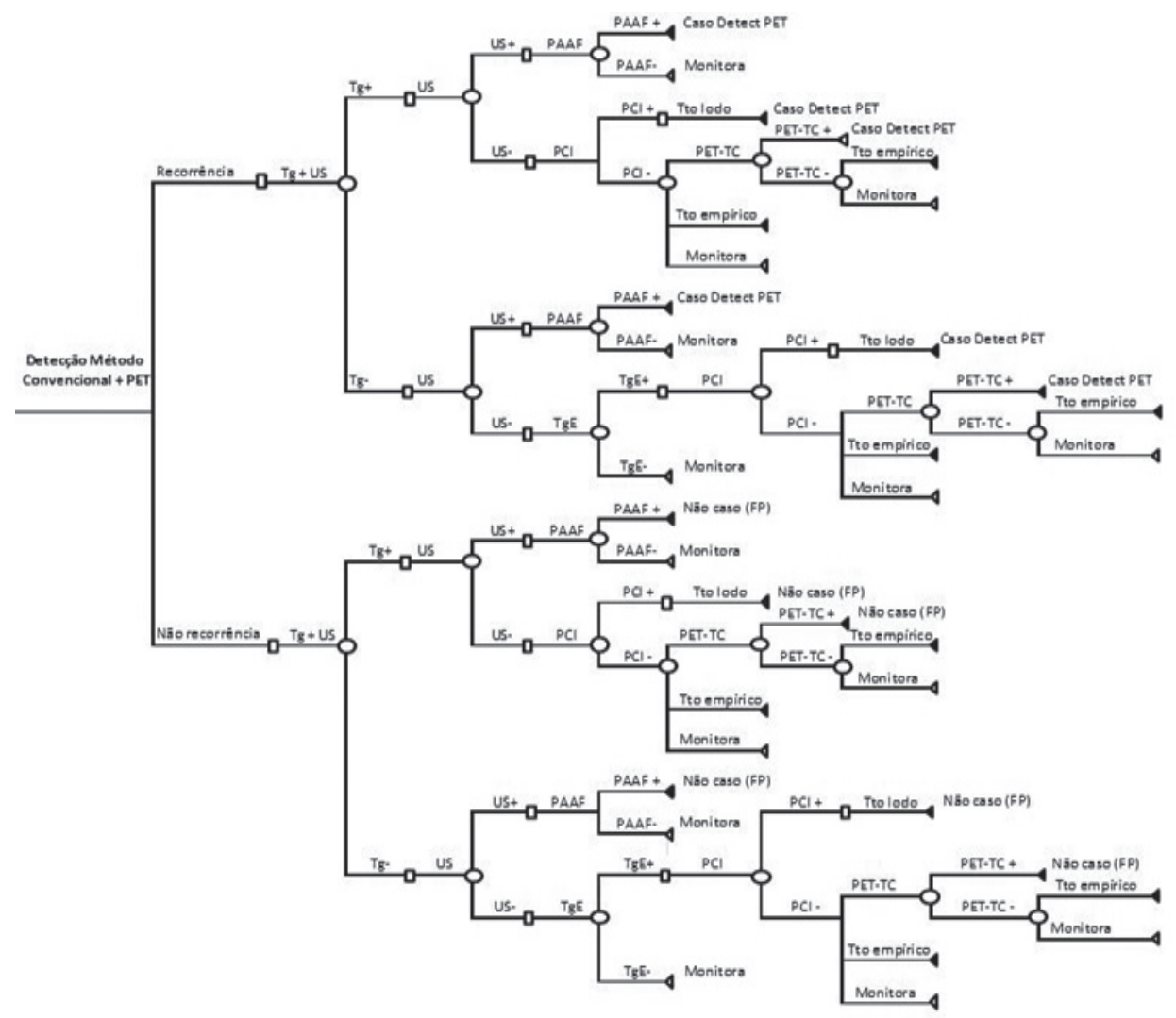

Legenda: ${ }^{18} \mathrm{FDG}$-PET/TC - Tomografia de emissão de pósitrons híbrida; FP - Falso-positivo; MC - Métodos Convencionais; PAAF - Punção aspirativa com agulha fina; PCI - Pesquisa de corpo inteiro; $\mathrm{Tg}$ - Tireoglobulina sérica suprimida; $\mathrm{TgE}$ - Tireoglobulina sérica estimulada; Tto - Tratamento com I ${ }^{131}$; US - Ultrassonografia

Fonte: elaboração própria.

\section{Parâmetros clínicos e de acurácia dos testes}

Informaçōes sobre a prevalência de doença recorrente pós-tratamento do CDT não foram identificadas em estudos nacionais. Utilizaram-se dados de prevalência relatados por Mazzaferri (1995; 2001), que resultaram do acompanhamento de uma grande coorte de 1.335 pacientes, de mais de 30 anos de seguimento (mediana de 16,7 anos). 
Dados os altos custos da PET-TC e ao fato de a tecnologia não estar presente nas tabelas de pagamento do SUS para esta indicação, a probabilidade de um indivíduo vir a fazer PET-TC após o resultado negativo da cintigrafia foi assumida como de $50 \%$ no caso de referência. $\mathrm{Na}$ análise de sensibilidade, esta foi variada entre $20 \%$ e $100 \%$, na tentativa de se estimar o benefício máximo em termos de casos adicionais de recorrência que pudessem ser detectados com a incorporação da tecnologia aos protocolos de manuseio.

A terapia empírica com iodo radioativo é muitas vezes utilizada em pacientes com forte suspeição de recorrência de CDT e técnicas de imagem negativas, mas não existe consenso a este respeito (CARBALLO; QUIROS, 2012) e sua indicação é frequentemente decidida caso a caso. A frequência deste uso na atualidade não foi identificada na literatura e assumiu-se como pressuposto, a partir da consulta a especialistas do INCA, que 30\% dos pacientes de alto risco com elevado grau de suspeição por Tg estimulada elevada ou crescentes poderiam vir a fazer uso da iodoterapia no caso de referência, com essa probabilidade de uso variada entre $10 \%$ e $50 \%$ na análise de sensibilidade.

A acurácia das tecnologias diagnósticas foi baseada na literatura (tabela 1). As medidas de acurácia utilizadas para a Tg suprimida e estimulada foram obtidas da metanálise de Eustatia-Rutten (2004) e, no caso da US cervical, optouse pelo emprego dos dados procedentes da metanálise de Wu (2012). Para a acurácia diagnóstica da $\mathrm{PCI}$, utilizaram-se dados obtidos de estudos prospectivos que envolviam populaçôes grandes e que realizaram o procedimento com TSH estimulado pela suspensão do hormônio tireoidiano exogenamente administrado (LUBIN, 1994; GALLOWITSCH, 1998; GRUNWALD, 1999). No caso da imagem PET, as medidas de acurácia utilizadas derivaram de metanálise empreendida especificamente com fins de subsidiar a realização desse estudo econômico com dados atualizados. ${ }^{2}$ Vinte estudos, publicados entre 1997 e 2012, foram incluídos. A sensibilidade e especificidade combinadas da PET-TC, usando modelo de efeitos randômicos, foram de, respectivamente, 93\% (IC 95\% de $84-97 \%$ ) e $81 \%$ (IC 95\% 69-90\%), com acurácia global medida pela área sob a curva SROC de 93\% (IC 95\% 91-95\%). 


\begin{tabular}{|c|c|c|c|}
\hline Variável & Caso Base & Intervalo & Referência \\
\hline \multicolumn{4}{|l|}{ Parâmetros clínicos } \\
\hline $\begin{array}{l}\text { Probabilidade de recorrência } \\
\text { de CDT }\end{array}$ & 0,2 & $0,07-0,24$ & $\begin{array}{l}\text { Brassard, 2011; } \\
\text { Mazzaferri, 1995; } \\
\text { Mazzaferri, } 2001\end{array}$ \\
\hline $\begin{array}{l}\text { Probabilidade de realização } \\
\text { de PET-TC }\end{array}$ & 0,5 & $0,2-1,0$ & Pressuposto \\
\hline $\begin{array}{l}\text { Probabilidade de tratamento } \\
\text { empírico com I }{ }^{131}\end{array}$ & 0,3 & $0,1-0,5$ & Pressuposto \\
\hline \multicolumn{4}{|l|}{ Acurácia dos testes } \\
\hline Sensibilidade Tg Suprimida & 0,78 & $0,3-0,83$ & Eustatia-Rutten, 2004 \\
\hline Especificidade Tg Suprimida & 0,98 & $0,96-1,0$ & Eustatia-Rutten, 2004 \\
\hline Sensibilidade Tg Estimulada & 0,96 & $0,84-0,97$ & $\begin{array}{l}\text { Eustatia-Rutten, 2004; } \\
\text { Gallowitsch, } 1998\end{array}$ \\
\hline $\begin{array}{l}\text { Especificidade } \mathrm{Tg} \\
\text { Estimulada }\end{array}$ & 0,95 & $0,88-1,0$ & Eustatia-Rutten, 2004 \\
\hline Sensibilidade US & 0,72 & $0,46-0,90$ & $\begin{array}{l}\text { Frasoldati, 2003, Wu, } \\
2012\end{array}$ \\
\hline Especificidade US & 0,98 & $0,84-1,0$ & Wu, 2012 \\
\hline Sensibilidade PAAF & 0,73 & $0,67-0,95$ & $\begin{array}{l}\text { Cunha, 2007; Jeon, 2009; } \\
\text { Baldini, } 2013\end{array}$ \\
\hline Especificidade PAAF & 0,82 & $0,55-0,96$ & $\begin{array}{l}\text { Cunha, 2007; Jeon, 2009; } \\
\text { Baldini, } 2013\end{array}$ \\
\hline Sensibilidade PCI & 0,55 & $0,43-0,77$ & $\begin{array}{l}\text { Lubin, 1994; Gallowitsch, } \\
\text { 1998; Grunwald, } 1999\end{array}$ \\
\hline Especificidade PCI & 0,98 & $0,96-1,0$ & $\begin{array}{l}\text { Lubin, 1994; Gallowitsch, } \\
\text { 1998; Grunwald, } 1999\end{array}$ \\
\hline Sensibilidade PET-TC & 0,93 & $0,84-0,97$ & Caetano, 2013 \\
\hline Especificidade PET-TC & 0,81 & $0,69-0,9$ & Caetano, 2013 \\
\hline
\end{tabular}

continua... 


\begin{tabular}{|c|c|c|c|}
\hline Variável & Caso Base & Intervalo & Referência \\
\hline \multicolumn{4}{|l|}{ Parâmetros de Custos } \\
\hline Tg Suprimida & 32,51 & $32,51-39,01$ & SIGTAP \\
\hline Tg Estimulada & 51,47 & $34,31-68,62$ & SIGTAP \\
\hline US cervical & 24,20 & $24,20-29,04$ & SIGTAP \\
\hline PAAF guiado US & 158,46 & $107,65-173,81$ & SIGTAP \\
\hline PCI & 338,70 & $338,70-406,44$ & SIGTAP \\
\hline $\begin{array}{l}\text { Tratamento empírico com } \\
\mathrm{I}^{131}\end{array}$ & $1.843,90$ & $\begin{array}{l}1.666,20- \\
2.182,90\end{array}$ & SIGTAP \\
\hline${ }^{18} \mathrm{FDG}-\mathrm{PET} / \mathrm{TC}$ & 2927,20 & $1.657,20-3.150,30$ & Caetano, 2014 \\
\hline Taxa de desconto (\%) & 5 & $0-10$ & BRASIL, 2009 \\
\hline
\end{tabular}

Legenda: $\mathrm{I}^{131}$ - Iodo $^{131}$; PAAF - Punção aspirativa com agulha fina; PCI - Pesquisa de corpo inteiro; PET-TC - Tomografia de emissão de pósitrons; Tg - Tireoglobulina sérica; US - Ultrassonografia. Fonte: elaboração própria.

\section{Custos}

A perspectiva adotada na avaliação foi a do SUS como financiador público da assistência à saúde, tendo sido considerados todos os custos médicos associados com a utilização dos métodos convencionais e ${ }^{18}$ FDG-PET-TC, estabelecidos conforme o desfecho em tela. Todos os custos foram expressos em reais de 2013.

Os custos diretos incluíram apenas os do diagnóstico da recorrência metastática do CDT. Nos casos de seguimento com reavaliação diagnóstica após um ano, foram considerados os custos correspondentes a, no máximo, mais nove ciclos de reavaliação, em função do horizonte temporal de dez anos estabelecido para o estudo.

Os custos das dosagens séricas de $\mathrm{Tg}$ suprimida e estimulada representaram um somatório do valor pago pelo teste e pela pesquisa de anticorpos antitireoglobulina, que todos os protocolos recomendam seja sempre realizada em concomitância. No caso da dosagem sérica de Tg estimulada, foi considerado ainda que o exame incluía a verificação prévia dos níveis séricos de TSH. Os custos da PAAF agregavam os custos da biópsia percutânea orientada por US, da citologia oncótica e avaliação pré-operatória mínima. 
Não foram considerados os custos com tratamentos, porque estes elementos não se encontravam incluídos na modelagem desenvolvida. A única exceção foi o tratamento empírico com iodoterapia, que correspondeu aos custos do tratamento empregando $200 \mathrm{mCi}$, dose mínima recomendada no protocolo brasileiro, dos exames sugeridos para o preparo para o tratamento, e da PCI diagnóstica pós-terapia.

Valores dos procedimentos diagnósticos e terapêuticos presentes nas tabelas de pagamento do SUS foram obtidos do Sistema de Gerenciamento da Tabela de Procedimentos, Medicamentos e OPM do SUS (SIGTAP), considerando vigência de junho/2013 (tabela 1). Os custos dos procedimentos de PET-TC, ainda não presentes nas tabelas de pagamento do SUS, foram estimados em estudo anterior pela técnica de microcustos, tomando por base a perspectiva de um provedor público de saúde e tendo o Instituto Nacional do Câncer (INCA) como lócus de estudo (CAETANO et al., 2014).

A análise foi realizada considerando o estadiamento convencional como a estratégia de base contra a qual se avaliou a adição da imagem PET no algoritmo diagnóstico e a eficiência comparativa das estratégias alternativas de detecção foi medida pela razão de custo-efetividade incremental (RCEI), definida como custo adicional da detecção dividido pelo benefício adicional em saúde, medido pelo número de casos adicionalmente identificados pela PET-TC. Dada a inexistência de limiar claro de custo-efetividade estabelecido no país para a incorporação de tecnologias, a estratégia mais custo-efetiva foi definida como aquela com menor RCEI.

Custos e benefícios foram descontados em 5\% (BRASIL, 2009), e variados entre 0 e $10 \%$ na análise de sensibilidade. Adicionalmente, também foram estimados os resultados da RCEI descontando apenas os custos, em função do desfecho intermediário escolhido no estudo.

Para examinar o impacto das incertezas nos parâmetros e pressupostos do modelo, foram realizadas análises de sensibilidade determinística univariada e probabilística, considerando os principais parâmetros clínicos, de acurácia das tecnologias, alguns itens de custo e a taxa de desconto. No caso da análise de sensibilidade probabilística, foi atribuída uma distribuição normal aos parâmetros clínicos e epidemiológicos e uma distribuição gama aos custos, sendo rodadas 1.000 simulações. 


\section{Resultados}

Com a estratégia convencional foram detectados 1.875 casos de recorrência ao final de dez anos de seguimento. O uso da ${ }^{18}$ FDG-PET-TC permitiu a deteç̧ão adicional de 13 casos (1.888 casos no total), representando um aumento de menos de $1 \%$, mas a um custo bastante significativo. Como resultado, o custo por cada caso adicional de recorrência detectado pela adição da tecnologia PET ao manuseio convencional situou-se em R \$ 477.633,05, em valores já descontados (tabela 2).

Tabela 2: Custo-efetividade das estratégias de detecção de CDT recorrente (em Reais de 2013)

\begin{tabular}{lccccc}
\hline $\begin{array}{l}\text { Estratégia de } \\
\text { Detecção }\end{array}$ & $\begin{array}{c}\text { Custos } \\
(\mathrm{em} \mathrm{R} \$)\end{array}$ & $\begin{array}{c}\Delta \text { custo } \\
(\mathrm{em} \mathrm{R} \text { ) }\end{array}$ & $\begin{array}{c}\text { Casos } \\
\text { detectados }\end{array}$ & $\begin{array}{c}\Delta \text { efeito } \\
\text { (casos } \\
\text { adicionais } \\
\text { detectados) }\end{array}$ & $\begin{array}{c}\text { RCEI } \\
\text { (custo em R \$ por } \\
\text { caso adicional } \\
\text { detectado) }\end{array}$ \\
\hline $\begin{array}{l}\text { Convencional } \\
\text { Convencional + } \\
\text { PET-TC }\end{array}$ & $11.715 .016,35$ & - & 1.875 & - & - \\
\hline
\end{tabular}

Legenda: ${ }^{18} \mathrm{FDG}-\mathrm{PET} / \mathrm{TC}$ - Tomografia de emissão de pósitrons híbrida com Tomografia computadorizada, utilizando radiofármaco ${ }^{18} \mathrm{FDG}$; RCEI - Razão de custo-efetividade incremental Fonte: elaboração própria.

\section{Análises de sensibilidade}

$\mathrm{Na}$ análise de sensibilidade determinística, foram explorados os impactos de parâmetros chaves sobre a medida de efetividade escolhida e sobre a razão de custoefetividade incremental da opção com adição da PET-TC em relação à estratégia convencional de deteç̧ão do CDT. Os parâmetros que produziram maior redução na RCEI foram os aumentos da especificidade da Tg estimulada e da taxa de desconto, e as reduçôes na sensibilidade da US e do custo do PET-TC. Por outro lado, reduções na taxa de desconto ou na probabilidade de recorrência metastática do CDT, bem como elevação nas sensibilidades da US e da PCI, produziriam aumento no custo por caso adicional detectado pela adição da PET-TC (figura 3). 

detectado pela adição da PET-TC ao manuseio convencional

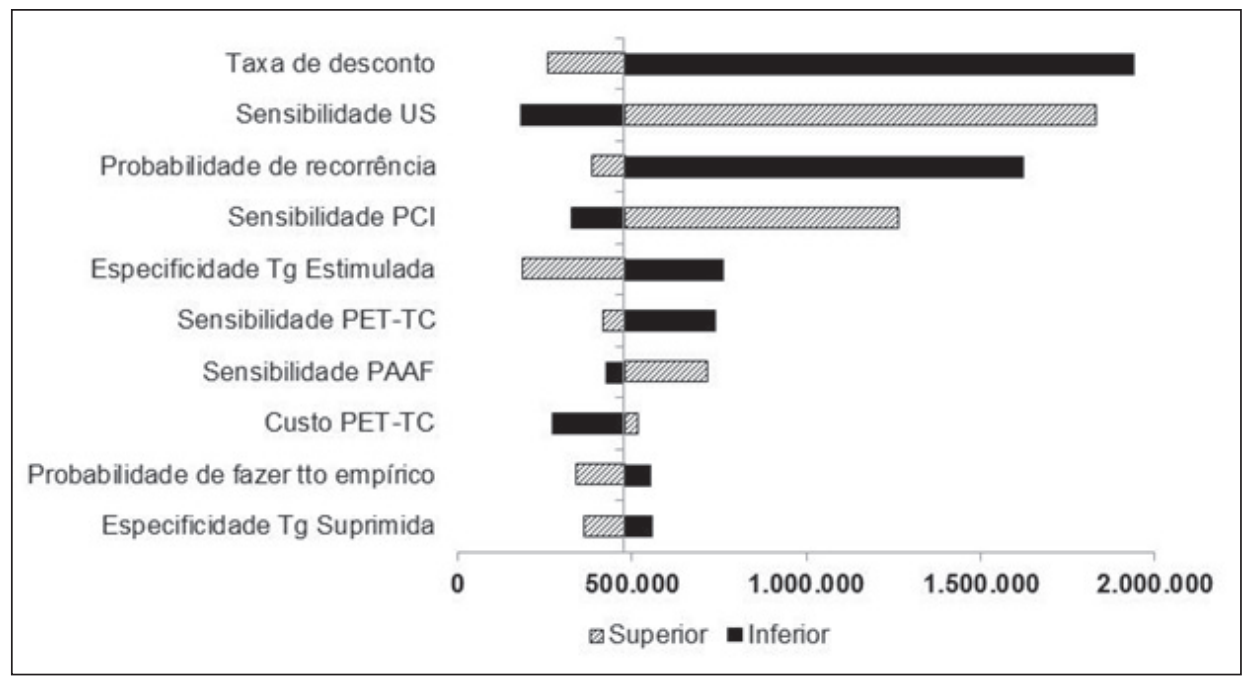

Legenda: PAAF - Punção aspirativa com agulha fina; PCI - Pesquisa de corpo inteiro; ${ }^{18}$ FDGPET/TC - Tomografia de emissão de pósitrons híbrida com Tomografia computadorizada, utilizando radiofármaco ${ }^{18} \mathrm{FDG}$; Tg - Tireoglobulina sérica; US - Ultrassonografia Fonte: elaboração própria.

Os resultados da análise adicional onde se aplicou a taxa de desconto de 5\% somente aos custos mostraram elevação dos valores por caso adicional detectado pela adição da PET-TC para $\mathrm{R} \$ 1.627 .436,88$.

Análises de sensibilidade probabilística envolvendo 1.000 simulações permitem visualizar que considerável incerteza ronda custos e potenciais benefícios da adição da PET-TC ao manuseio convencional do seguimento do CDT, tanto sobre a extensão e existência de custos incrementais (mostrados no plano vertical), quanto sobre os resultados em saúde, em termos de casos adicionais detectados (plano horizontal), em particular sobre estes últimos, que se encontram mais dispersos que os primeiros (figura 4). 

convencionais na detecção de recorrência de CDT

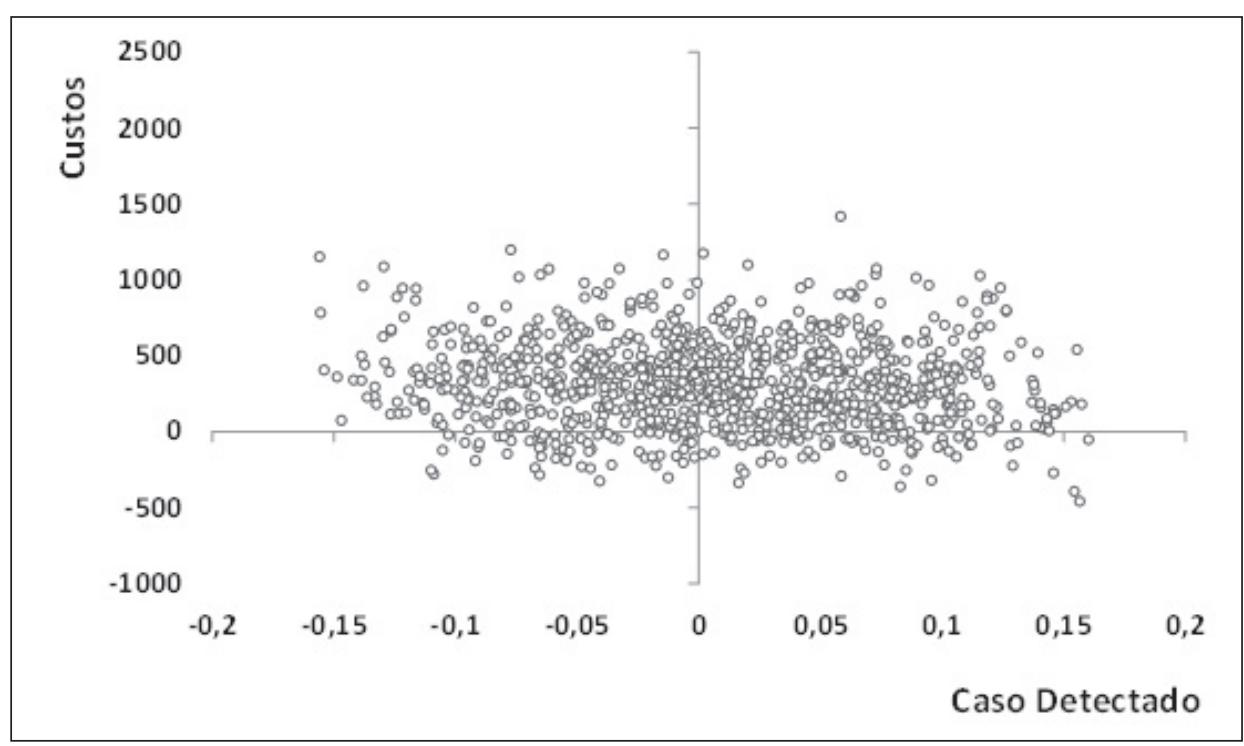

Legenda: PET-TC - Tomografia de emissão de pósitrons conjugada à tomografia computadorizada; CDT - Câncer diferenciado de tireoide

Fonte: elaboração própria.

\section{Discussão}

A despeito da grande maioria de casos de câncer diferenciado de tireoide ter natureza indolente e uma história natural favorável, uma proporção de até 30 a $35 \%$ dos casos apresentaram recorrência, usualmente nos primeiros dez anos do diagnóstico. Assim, a vigilância após o tratamento inicial é fundamental para a detecção de recorrência e delineamento de estratégias terapêuticas que reduzam o risco de morte pela doença. $\mathrm{O}$ seguimento desses pacientes tem se alterado nos últimos anos, com um uso mais intensivo da US, o reconhecimento das limitaçôes diagnósticas da PCI e o surgimento de tratamentos alternativos para casos mais avançados da neoplasia (TUTLE, 2008). A PET-TC é advogada como sendo útil nesse acompanhamento, particularmente nos casos de tumores recorrentes sem avidez por iodo. Entretanto, o nicho de oportunidade para seu uso é bastante seletivo e seus custos elevados dificultam o acesso dos pacientes que possam porventura se beneficiar de sua acurácia nessa condição. 
Oferecer uma nova tecnologia de alto custo, como é o caso do PET scan, traz importantes custos financeiros para o sistema, que podem ser contrabalanceados ou não por melhorias nos desfechos dos pacientes. Preocupações com o uso de recursos sempre escassos na saúde não são uma prerrogativa brasileira. Entretanto, chama atenção a total ausência, na literatura pesquisada, de estudos de avaliação econômica relativos ao uso da imagem PET no câncer de tireoide, independentemente de indicação ou tipo histológico. Publicações recentes de Buck (2010) e Saif (2010), que revisaram estudos econômicos relativos à PET e PETTC em aplicações oncológicas diversas, também não fazem menção a qualquer avaliação desse tipo na neoplasia. É possível que esta lacuna verificada decorra da frequência relativamente baixa do câncer tireoidiano e do espaço de uso clínico, bastante restrito e específico, que é reservado à imagem PET nos algoritmos diagnósticos nessa neoplasia. Por outro lado, este vazio informacional reforça a importância dos resultados deste estudo em prover informação potencialmente relevante para os tomadores de decisão.

O trabalho evidenciou que a adição da ${ }^{18}$ FDG-PET-TC no seguimento do CDT é efetiva em comparação à estratégia convencionalmente utilizada. Isto se faz, contudo, a custos muito elevados, superior a 475 mil reais por caso adicional detectado. O Brasil não possui um limiar formal de relação de custo-efetividade incremental (ICER) aceitável para incorporação de tecnologias. A Organização Mundial da Saúde recomenda, nessa situação, a utilização de valores até três vezes o PIB per capita do país como limite para se considerar uma intervenção custo-efetiva, mas esta indicação toma por base medidas de desfecho finalístico, como anos de vida salvos ou anos de vida ganhos ajustados por qualidade (WHO, 2003). Mesmo considerando esta restrição, o valor de quase 500 mil reais representa quase 20 vezes o valor do PIB per capita brasileiro em 2013, dificultando significativamente sua incorporação e uso mais disseminado, especialmente no SUS.

Fica evidente também, a partir da análise de sensibilidade probabilística realizada, que existe elevado grau de incerteza em relação tanto aos diferenciais de custo como de efetividade relacionados com a adição da tecnologia. A nuvem de pontos obtida na análise apresenta-se muito dispersa pelos quadrantes do plano de custo-efetividade; não há predomínio de sua distribuição em relação a uma maior efetividade, mas modo geral existe tendência marcada de um custo mais elevado associado a esta adição. 
As análises determinísticas assinalam um conjunto de parâmetros com potencial de responder por essas incertezas. Assim, se a probabilidade de recorrência do CDT for mais baixa que o estimado no caso de referência, o custo por caso detectado se eleva, chegando a $\mathrm{R} \$ 1.623 .264,76$ para prevalência em dez anos de cerca de 7\%. A literatura cita percentuais de recorrência que variam de 5 a 35\% dos casos (MAZZAFERRI et al., 1995; MAZZAFERRI et al., 2001; JOHNSON; TUBLIN, 2008). Foi utilizada uma taxa de prevalência em dez anos de $20 \%$, oriunda de trabalhos que acompanharam grandes coortes, por longo tempo, mas cujo acompanhamento se iniciou muitas vezes nos anos 60 e 70. Desde então, as práticas de detecção e de tratamento se alteraram significativamente. A incidência de CDT se elevou bastante em alguns países nos últimos 30 anos, embora o tamanho do tumor ao diagnóstico venha diminuindo de forma constante, o que pode decorrer de possíveis melhorias na detecção pelo uso mais frequente de imagem de melhor qualidade (DAVIES; WELCH, 2006). Sabe-se também que as terapêuticas cirúrgica e clínica inicial do CDT têm efeitos no longo prazo que independem de outros fatores de risco como a idade do paciente e diagnóstico histológico (MAZZAFERRI; JHIANG, 1994). Assim, se mais casos são detectados em estadios mais precoces, com melhor chance de intervenção terapêutica eficaz, o risco posterior de recorrência pode ser alterado, produzindo cenário ainda menos favorável à adição da PET-TC.

Outro elemento de impacto significativo nos resultados apresentados é a performance diagnóstica da US, que tanto pode elevar quanto reduzir os custos por caso adicional detectado pela ${ }^{18}$ FDG-PET-TC. Nos últimos anos, esta técnica de baixo custo e acesso fácil (e o uso da PAAF guiado pela mesma) tem emergido como a modalidade de imagem dominante tanto para o diagnóstico quanto, posteriormente, para o seguimento dos casos. Progressos técnicos, como melhor resolução de imagem e US doppler colorido, vêm permitindo avanços na identificação e localização de lesões cervicais progressivamente menores e facilitado amostras tissulares para confirmação (STULAK et al., 2006). Aumento na sensibilidade da US eleva a possibilidade de casos detectados pela estratégia convencional de seguimento e penaliza os valores de RCEI relacionados à adição da PET, na medida em que reduz o número de casos adicionais potencialmente detectados por esta última. Por outro lado, é reconhecida a grande variabilidade na sensibilidade da tecnologia dependente da expertise do operador (WU et al., 2012), 
e seu uso limitado à região cervical não permite detecção de lesões recorrentes à

distância, mantendo a necessidade de utilização de outras técnicas de imagem, nos casos de marcadores séricos positivos com exame ultrassonográfico negativo.

Uma maior sensibilidade da cintigrafia diagnóstica com I ${ }^{131}$ também impacta negativamente na RCEI da estratégia com PET, dada sua indicação prioritária para os casos negativos à PCI. A RCEI da adição da PET pode chegar a R\$ 1.262.602,98, com sensibilidade da PCI de 77\%. A favor do emprego da PCI no seguimento das recorrências estão seu baixo custo, a ampla disseminação nos serviços e a facilidade de acesso, não presentes para a PET-TC. Entretanto, o uso mais disseminado das dosagens de $\mathrm{Tg}$ e da US, bem como o reconhecimento crescente das limitações de sensibilidade da PCI, tem determinado decréscimo de sua relevância clínica e contribuído para limitar progressivamente sua utilização a situações específicas (ATA, 2009), como a detecção de metástases distantes em pacientes com marcador sérico elevado e imagem negativa.

Cabe chamar atenção para o efeito produzido pelos custos da PET-TC na RCEI. O PET scan ainda não possui grande difusão no país e apenas recentemente (abril de 2014) sua cobertura foi incluída no SUS, mas para indicações diferentes da examinada. Até a elaboração deste trabalho, ainda não havia valores definidos para o procedimento nas tabelas de pagamento do sistema público, o que determinou que os dados utilizados na modelagem derivassem de estudo de microcustos específico. Mesmo empregando o limite inferior do intervalo utilizado ( $R$ \$ 1.657,20), que é menor que o valor de $R$ \$2.107,22 estabelecido em dezembro de 2014 para o pagamento do procedimento PET na tabela no SUS para as indicações já aprovadas (BRASIL, 2014d), a redução dos custos por caso adicional detectado com a estratégia PET-TC cairia para R $\$ 273.196,14$, ainda bastante elevado para o cenário brasileiro. Embora reconhecendo que diversos itens relacionados à tecnologia PET-TC se alteraram nos últimos tempos, como o preço dos equipamentos e do radiofármaco - que tem decaído à medida que a tecnologia entra em uma fase de maior estabilização de design e que aumenta a produção comercial privada do radioisótopo -, é bastante razoável supor que muito dificilmente o custo do procedimento caia a valores ainda menores que o simulado no limite inferior mencionado.

Dada a relevância da taxa de desconto nos resultados da RCEI e as controvérsias entre os autores sobre a decisão entre aplicar ou não aplicar o desconto nos 
resultados em saúde (BRASIL, 2009), foi examinado o impacto da aplicação do desconto restrito apenas a custos. Nessa situação, o custo por caso detectado ascenderia a valores 3,4 vezes os encontrados com a aplicação a ambos, porque a PET-TC detecta a grande maioria dos casos logo no primeiro ano dos dez anos de seguimento, dada a sua acurácia.

Tecnologias diagnósticas, como é o caso do PET scan, são sempre desafiadoras em termos de sua avaliação. No caso específico do CDT, os processos de manuseio diagnóstico propostos para seu acompanhamento, nos últimos anos, têm se pautado por uma tentativa de "personalizar" o seguimento segundo riscos estratificados de recorrência (TUTTLE; LEBOEUF, 2008). Esses riscos são definidos não apenas em função das características iniciais dos tumores tireoidianos e dos pacientes, mas também da resposta ao tratamento e seus resultados evolutivos (ATA, 2009).

A complexidade desses processos agrega significativas dificuldades para as modelagens, ainda mais quando se reconhece que modelos são sempre simplificações da realidade. Seu uso, contudo, permite que se obtenha uma visão mais clara (e de manuseio factível, inclusive no que concerne aos custos de obtenção dos dados) do problema como um todo e que se concentre em seus aspectos mais relevantes. A capacidade dos modelos de extrapolar, adaptar e integrar dados de diferentes fontes de forma transparente torna essa ferramenta especialmente indicada em estudos de avaliação econômica, pois revelam conexôes entre os pressupostos e dados e os resultados do modelo, ajudando a identificar a via preferível entre duas possibilidades de intervenção (RODRÍGUEZ BARRIOS, 2004).

Pode-se argumentar que o uso de modelagem, em geral e neste caso em particular, acaba não levando em conta algumas nuanças da doença em questão, de certo modo tão complexa. Porém, quanto mais complicado o modelo construído, mais difícil se torna obter dados suficientemente detalhados para sua alimentação. Desta forma, tentou-se utilizar um modelo relativamente simplificado, mas que incluísse e considerasse todos os aspectos mais relevantes do manejo da neoplasia presentes nos protocolos mais atualizados, inclusive o brasileiro. Ao mesmo tempo, extensa gama de variáveis e pressupostos foram testados nas análises de sensibilidade, ajudando na compreensão das incertezas e de seus impactos nos resultados obtidos, e facilitando sua apropriação pelos decisores e outros interessados. 
A dificuldade informacional encontrada para a identificação de alguns parâmetros é outra limitação a ser mencionada. Esta dificuldade determinou decisão de não se incluir algumas tecnologias referidas nos protocolos ou de se trabalhar, em alguns casos, com pressupostos, arbitrados em função das indefinições encontradas na literatura.

A falta de informaçôes sobre a acurácia da pesquisa de autoanticorpos antiTg levou ao pressuposto de que todos os pacientes da coorte hipotética tinham sido testados a cada dosagem de $\mathrm{Tg}$, conforme recomendam os protocolos, e eram negativos, permitindo assim a agregação dos custos do procedimento nos braços pertinentes.

A mesma dificuldade de disponibilidade dos dados se aplica à acurácia das biópsias confirmatórias dos resultados positivos à PET, que é variável segundo a localização dos achados de imagem. Esta limitação foi manuseada por um desenho analítico que partia do status de doença recorrente, definida a partir das prevalências identificadas na literatura. Se, por um lado, isto permitiu separar os resultados positivos da PET-TC em verdadeiro-positivos e falsopositivos independentemente da biópsia, por outro implicou que os custos dos procedimentos de biópsia não tenham sido agregados nessa estratégia, o que em última instância significa que os valores de custo por caso adicional detectado seriam ainda mais elevados.

Dados confiáveis sobre o desempenho diagnóstico da TC de tórax para detecção de recorrência não foram encontrados, mas a utilização da PET-TC permite identificar esses casos pelo componente anatômico da tecnologia híbrida. Se considerarmos que uma parcela desses casos poderia ter sido diagnosticada pelo uso prévio apenas da TC torácica, o número de casos que seriam detectados pela imagem PET seria ainda menor, com as consequentes repercussões negativas na RCEI da estratégia.

Foi feito ainda o pressuposto de que apenas $50 \%$ dos pacientes com resultados de cintigrafia negativa seriam submetidos ao PET-TC, com essa probabilidade variada na análise de sensibilidade entre 20 e 100\%. Mesmo em países onde a tecnologia encontra-se presente nas tabelas de reembolso, há restrições e regulação ao seu uso para o câncer de tireoide. Nos EUA, por exemplo, a cobertura da PET e PET-TC pelo Medicare/Medicaid no seguimento pós-tratamento e definição de condutas terapêuticas posteriores está restrita a cânceres tireoidianos de 
origem folicular previamente tratados com tireoidectomia e ablação, que apresentem Tg sérica superior a 10ng/ml e PCI negativa (CENTERS..., 2006a). Todas as demais indicações da PET no seguimento do CDT apenas são cobertas se vinculadas a iniciativas do chamado Coverage with Evidence Development, no qual a cobertura é condicionada à coleta de evidências adicionais sobre a efetividade das tecnologias (CENTERS..., 2006b).

O tratamento dos casos de recorrência detectados não foi contemplado no estudo, que optou por uma unidade de desfecho mais simples, centrada nos casos detectados por cada uma das estratégias modeladas. A opção por não incluir os procedimentos subsequentes decorreu da variedade de tratamentos existentes, que são função inclusive da localização da recorrência e das condições individuais de cada paciente. A única exceção foi a inclusão do tratamento empírico com $\mathrm{I}^{131}$ como um nó terminal em alguns ramos das árvores de decisão, porque um conjunto de indivíduos, que possuem resultados negativos com a PCI ou a PETTC (realizada após PCI negativa), saem da coorte por esse caminho.

Por fim, deve ser discutida a medida de efetividade selecionada: caso detectado pelas estratégias de seguimento do CDT. Este desfecho intermediário não pode ser facilmente traduzido em resultados finalísticos em saúde, como mortes evitadas, ganho em sobrevida, ou qualidade de vida. Entretanto, o conhecimento acurado dos casos de recorrência é condição para o estabelecimento posterior de condutas terapêuticas que possam impactar significativamente na mortalidade, morbidade e qualidade de vida dos pacientes. Dados que permitissem a alimentação do modelo com os resultados em saúde das diferentes estratégias diagnósticas e terapêuticas não foram encontrados em estudos nacionais ou internacionais, inviabilizando sua aplicação nesse momento. O modelo desenvolvido é passível de melhorias e adaptações futuras que permitam incorporar a sobrevida, qualidade de vida ou as preferências dos pacientes, reiterando-se a importância de investigações posteriores que utilizem estes desfechos.

\section{Conclusões}

O estudo apontou que a incorporação do uso da PET-TC na detecção de recorrências metastáticas do CDT, em pacientes negativos à $\mathrm{PCI}$, promove a identificação de casos adicionais em relação à estratégia convencional. No 
entanto, os custos agregados são significativos e os valores por caso adicional detectado bastante elevados e superiores a $\mathrm{R} \$ 477.000,00 .^{3}$

\section{Referências}

AMERICAN Thyroid Association (ATA) Guidelines Taskforce on Thyroid Nodules and Differentiated Thyroid Cancer et al. Revised American Thyroid Association management guidelines for patients with thyroid nodules and differentiated thyroid cancer. Thyroid, [s.1.], v. 19, n. 11, p. 1167-1214, Nov. 2009.

BALDINI, E. et al. Cervical lymph node metastases from thyroid cancer: does thyroglobulin and calcitonin measurement in fine needle aspirates improve the diagnostic value of cytology? BMC Clin Pathol, [s.1.], v. 13, n. 7, p. 1-8, Feb. 2013.

BRASIL. Emenda Constitucional no 49, de 08 de fevereiro de 2006. Altera a redação da alínea b e acrescenta alínea e ao inciso xxiii do caput do art. 21 e altera a redação do inciso V do caput do art. 177 da Constituição Federal para excluir do monopólio da União a produção, comercialização e utilização de radioisótopos de meia-vida curta, para usos médicos, agrícolas e industriais. Brasília: Diário Oficial [da] República Federativa do Brasil, fev. 2006, Seção 1, n. 26, p. 1.

. Portaria no 7 de 22 de abril de 2014. Torna pública a decisão de incorporar o PETCT no estadiamento clínico do câncer de pulmão de células não-pequenas potencialmente ressecável no Sistema Único de Saúde - SUS. Brasília: Diário Oficial [da] República Federativa do Brasil, abr. 2014 [a], Seção 1, n. 76, p.78.

. Portaria no 8 de 22 de abril de 2014. Torna pública a decisão de incorporar o PETCT na detecção de metástase de câncer colorretal, exclusivamente hepática e potencialmente ressecável no Sistema Único de Saúde - SUS. Brasília: Diário Oficial [da] República Federativa do Brasil, abr. 2014 [b], Seção 1, n. 76, p.78.

. Portaria no 9 de 22 de abril de 2014. Torna pública a decisão de incorporar o PET-CT no estadiamento e avaliação da resposta ao tratamento do linfoma de Hodgkin e linfoma não Hodgkin no Sistema Único de Saúde - SUS. Brasília: Diário Oficial [da] República Federativa do Brasil, abr. 2014 [c], Seção 1, n. 76, p.79.

. Portaria no 1.340 de 01 de dezembro de 2014. Inclui procedimento PET-CT na Tabela de Procedimentos, Medicamentos, Órteses/Próteses e Materiais Especiais do SUS. Diário Oficial da Uniāo, Brasília, 2014. (BRASIL, 2014e) Diário Oficial [da] República Federativa do Brasil, dez. 2014 [d], Seção 1, n. 233, p.32.

- Ministério da Saúde. Instituto Nacional de Câncer. Estimativa 2016/2017: Incidência de Câncer no Brasil. Rio de Janeiro: INCA, 2015. Disponível em: http://www. inca.gov.br/wcm/dncc/2015/dados-apresentados.pdf>. Acesso em: 19 dez 2015. 

. Ministério da Saúde. Secretaria de Assistência à Saúde. Portaria nº 7, de 3 de janeiro de 2014. Aprova o Protocolo Clínico e Diretrizes Terapêuticas do Carcinoma Diferenciado da Tireoide. Brasília: Diário Oficial [da] República Federativa do Brasil, jan. 2014 [e], Seção 1, n. 4, p. 20-25.

- Ministério da Saúde. Secretaria de Ciência, Tecnologia e Insumos Estratégicos. Departamento de Ciência e Tecnologia. Diretrizes Metodológicas: estudos de avaliação econômica de tecnologias em saúde. Brasília: Ministério da Saúde, 2009.

BRASSARD, M. et al. Long-term follow-up of patients with papillary and follicular thyroid cancer: a prospective study on 715 patients. J Clin Endocrinol Metab., [Chevy Chase], v.96, i.5, p.1352-1359, May 2011.

BROSA, M. et al. Principios, métodos y aplicaciones del análisis del impacto presupuestario en el sector sanitario. Pharmacoeconomics - Spanish Research Articles, [Madrid], v. 2, i. 2, p. 64-78, Apr. 2005.

BUCK, A. K. et al. Economic evaluation of PET and PET/CT in oncology: evidence and methodologic approaches. J Nucl Med Technol., [s.l.], v. 38, n. 1, p. 6-17, Mar. 2010.

CAETANO, R. et al. Análise dos custos do procedimento PET-TC com 18 F-FDG na perspectiva do SUS provedor: estudo em uma unidade pública de saúde do Rio de Janeiro, Brasil. Cad. Saúde Pública, Rio de Janeiro, v. 30, n. 2, p. 379-392, fev. 2014.

Custo-efetividade da tomografia de emissão de pósitrons (PET) com F18-fluro-2desoxi-D-glicose (18F-FDG) nas seguintes aplicaçôes em oncologia: câncer de pulmão de células não pequenas, câncer de tireoide e câncer de cólon e reto. Projeto CNPq 564797/2010-3. Relatório de Pesquisa. Rio de Janeiro: Instituto de Medicina Social, jul. 2013.

CARBALLO, M.; QUIROS, R. M. To treat or not to treat: the role of adjuvant radioiodine therapy in thyroid cancer patients. J Oncol., [New York], v. 2012; n. 707156, Nov. 2012.

CENTERS for Medicare and Medicaid Services (CMS). National coverage determination (NCD) for FDG PET for Thyroid Cancer (220.6.11). [Baltimore]: CMS, 2006. Disponível in: <http://www.cms.gov/medicare-coverage-database/details/ncd-details.aspx?NCDId=3 $02 \&$ ncdver $=2 \&$ NCAId $=70 \&$ NcaName $=$ Positron + Emission + Tomography $+($ FDG $)+$ for $+\mathrm{T}$ hyroid + Cancer $\&$ IsPopup $=y \& b c=$ AAAAAAAACAAAAA\%3D\%3D\&>. Acesso em: 08 jul. 2013.

National coverage determinations with data collection as a condition of coverage: coverage with evidence development. [Baltimore]: CMS, 2006. Disponível em: <http:// www.cms.gov/Medicare/Coverage/DeterminationProcess/Downloads/ced.pdf>. Acesso em: 08 jul. 2013.

CUNHA, N. et al. Thyroglobulin detection in fine-needle aspirates of cervical lymph nodes: a technique for the diagnosis of metastatic differentiated thyroid cancer. Eur J Endocrinol., [s.1.], v. 157, n. 1, p. 101-107, Jul. 2007. 
DAVIES, L.; WELCH, H. G. Increasing incidence of thyroid cancer in the United States: 1973-2002. JAMA, [s.1.], v. 295, n. 18, p. 2164-2167, May 2006.

EUSTATIA-RUTTEN, C. F. et al. Diagnostic value of serum thyroglobulin measurements in the follow-up of differentiated thyroid carcinoma, a structured meta-analysis. Clin Endocrinol, Oxford, v. 61, n. 1, p. 61-74, Jul. 2004.

FEINE, U. et al. Fluorine-18-FDG and iodine-131-iodide uptake in thyroid cancer. $J$ Nucl Med., Reston, v. 37, n. 9, p. 1468-1472, Sep. 1996.

FRASOLDATI, A. et al. Role of thyroglobulin measurement in fine-needle aspiration biopsies of cervical lymph nodes in patients with differentiated thyroid cancer. Thyroid, [s.1.], v. 9, n. 2, p. 105-111, Feb. 1999.

GALLOWITSCH, H. J. et al. Thyroglobulin and low-dose iodine-131 and technetium99m-tetrofosmin whole-body scintigraphy in differentiated thyroid carcinoma. J Nucl Med., Reston, v. 39, n. 5, p. 870-875, May 1998.

GRÜNWALD, F. et al. Fluorine-18 fluorodeoxyglucose positron emission tomography in thyroid cancer: results of a multicentre study. Eur J Nucl Med., [s.1.], v. 26, n. 12, p. 15471552, Dec. 1999.

HAY, I. D. et al. Papillary thyroid carcinoma managed at the Mayo Clinic during six decades (1940-1999): temporal trends in initial therapy and long-term outcome in 2444 consecutively treated patients. World J Surg., [s.1.], v. 26, n. 8, p. 879-885, Aug. 2002.

JEON, S. J. et al. Diagnostic benefit of thyroglobulin measurement in fine-needle aspiration for diagnosing metastatic cervical lymph nodes from papillary thyroid cancer: correlations with US features. Korean J Radiol., [s.l.], v. 10, n. 2, p. 106-111, Mar. 2009.

JOENSUU, H.; AHONEN, A. Imaging of metastases of thyroid carcinoma with fluorine-18 fluorodeoxyglucose. J Nucl Med., Reston, v. 28, n. 5, p. 910-914, May 1987.

JOHNSON, N. A.; TUBLIN, M. E. Postoperative surveillance of differentiated thyroid carcinoma: rationale, techniques, and controversies. Radiology, [Oak Brook], v. 249, n.2, p. 429-444, Nov. 2008.

LANG, B. H.; LAW, T. T. The role of 18F-fluorodeoxyglucose positron emission tomography in thyroid neoplasms. Oncologist, Durham, v.16, n.4, p. 458-466, Apr. 2011.

LIND, P. et al. 18F-FDG-PET in the follow-up of thyroid cancer. Acta Med Austriaca, [s.1.], v. 30, n. 1, p. 17-21, Jan 2003.

LIND, P.; KOHLFURST, S. Respective roles of thyroglobulin, radioiodine imaging, and positron emission tomography in the assessment of thyroid cancer. Semin Nucl Med., [s.1.], v. 36, n. 3, p. 194-205, Jul. 2006.

LUBIN, E. et al. Serum thyroglobulin and iodine-131 whole-body scan in the diagnosis and assessment of treatment for metastatic differentiated thyroid carcinoma. J Nucl Med., Reston, v. 35, n. 2, p. 257-262, Feb. 1994. 
MA, C.; XIE, J.; KUANG, A. Is empiric 131I therapy justified for patients with positive thyroglobulin and negative 131I whole-body scanning results? J Nucl Med., Reston, v. 46, n. 7, p. 1164-1170, Jul. 2005.

MAZZAFERRI, E. L.; JHIANG, S. M. Differentiated thyroid cancer long-term impact of initial therapy. Trans Am Clin Climatol Assoc., San Antonio, v. 106, p. 151-170, 1995.

MAZZAFERRI, E. L. Long-term impact of initial surgical and medical therapy on papillary and follicular thyroid cancer. Am J Med., Tucson, v. 97, i. 5, p. 418-428, Nov. 1994.

MAZZAFERRI, E. L.; KLOOS, R. T. Clinical review 128: Current approaches to primary therapy for papillary and follicular thyroid cancer. J Clin Endocrinol Metab., [Chevy Chase], v. 86, n. 4, p. 1447-1463, Apr. 2001.

MILAS, Z.; SHIN, J.; MILAS, M. New guidelines for the management of thyroid nodules and differentiated thyroid cancer. Minerva Endocrinol., [s.1.], v. 36, n. 1, p. 53-70, Mar. 2011.

NAHAS, Z. et al. The role of positron emission tomography/computed tomography in the management of recurrent papillary thyroid carcinoma. Laryngoscope, [Omaha], v. 115, n. 2, p. 237-243, Feb. 2005.

PACINI, F. et al. Thyroid cancer: ESMO Clinical Practice Guidelines for diagnosis, treatment and follow-up. Ann Oncol., [s.l.], v. 23, suppl. 7, p. vii110-vii9, Oct. 2012.

RIEMANN, B. et al. Diagnostic value and therapeutic impact of (18)F-FDG-PET-CT in differentiated thyroid cancer. Results of a German multicentre study. Nuklearmedizin, [s.l.], v. 52, n. 1, p.1-6, 2013.

RODRÍGUEZ BARRIOS, J.M. Papel de los modelos en las evaluaciones económicas en el campo sanitário. Farm Hosp., [s.l.], v. 28, n. 4, p. 231-242, Jul. 2004.

ROSENBAUM, M. A.; MCHENRY, C. R. Contemporary management of papillary carcinoma of the thyroid gland. Expert Rev Anticancer Ther., [s.1.], v. 9, n. 3, p. 317-329, Mar. 2009.

SAIF, M. W. et al. Role and cost effectiveness of PET/CT in management of patients with cancer. Yale J Biol Med., New Haven, v. 83, n. 2, p. 53-65, Jun. 2010.

SHOUP, M. et al. Prognostic indicators of outcomes in patients with distant metastases from differentiated thyroid carcinoma. J Am Coll Surg., Chicago, v. 197, n. 2, p. 191-197, Aug. 2003.

SIBILLE, L. et al. Impact de la TEP/TDM au 18F-FDG dans la prise en charge des patients atteints de cancer thyrö̈dien différencié. Médecine Nucléaire, [s.l.], v. 34, n. 2, p. 78-87, Feb. 2010.

STULAK, J. M. et al. Value of preoperative ultrasonography in the surgical management of initial and reoperative thyroid cancer. Arch Surg., [s.1.], v. 141, n. 5, p. 489-496, May 2006. TUTTLE, R. M. et al. Estimating risk of recurrence in differentiated thyroid cancer after total thyroidectomy and radioactive iodine remnant ablation: using response to therapy 
variables to modify the initial risk estimates predicted by the new American Thyroid Association staging system. Thyroid, [s.l.], v. 20, n. 12, p. 1341-1349, Dec. 2010.

TUTTLE, R. M.; LEBOEUF, R.; SHAHA, A. R. Medical management of thyroid cancer: a risk adapted approach. J Surg Oncol., [s.1.], v. 97, i. 8, p. 712-716, Jun. 2008.

VIANNA, C. M. M.; CAETANO, R. Avaliaçôes econômicas como um instrumento no processo de incorporação tecnológica em saúde. Cad Saúde Colet., Rio de Janeiro, v. 13, n. 3, p. 747-766, jul.-set. 2005.

WORLD HEALTH ORGANIZATION. Making choices in health: WHO guide to cost-effectiveness analysis. Geneva, World Health Organization 2003. Disponível em: $<$ http://www.who.int/choice/publications/p_2003_generalised_cea.pdf?ua=1>. Acesso em: 08 jun 2015.

WU, L. M. et al. The accuracy of ultrasonography in the preoperative diagnosis of cervical lymph node metastasis in patients with papillary thyroid carcinoma: A meta-analysis. Eur J Radiol., [s.1.], v. 81, i. 8, p. 1798-1805, Aug. 2012.

\section{Notas}

${ }^{1}$ A diretriz da Associação Americana de Tireoide adota uma estratificação que classifica o risco de recorrência dos pacientes em baixo, intermediário e alto. São considerados pacientes com alto risco de recorrência: idade inferior a 20 anos ou superior a 60 anos; tamanho do tumor primário superior a $4 \mathrm{~cm}$; ressecção tumoral incompleta na cirurgia; invasão macroscópica extratireoidiana ou invasão vascular; envolvimento ganglionar ou presença de metástases distantes no diagnóstico (ATA, 2009).

${ }^{2}$ Esta metanálise fez parte da pesquisa "Custo-efetividade da tomografia de emissão de pósitrons com F18-fluro-2-desoxi-D-glicose (18F-FDG-PET) nas seguintes aplicações em oncologia: câncer de pulmão de células não pequenas, câncer de tireoide e câncer de cólon e reto", realizada para o Ministério da Saúde (Projeto CNPq 564797/2010-3) e finalizada em julho de 2013. O artigo em questão, intitulado "Accuracy of PET and PET-CT in the Detection of Differentiated Thyroid Cancer Recurrence with Negative ${ }^{131} I$ Whole Body Scan Results: A Meta-Analysis", submetido à revista Head \& Neck em dezembro de 2013, ainda se encontra em avaliação.

${ }^{3}$ R. Caetano participou da concepção e projeto do estudo, concepção e desenvolvimento do modelo analítico de decisão, interpretação dos dados, redação do artigo e aprovação da versão final a ser publicada. A. N. Biz participou do desenvolvimento do modelo analítico de decisão, coleta dos dados de custo, redação e revisão crítica do artigo e aprovação da versão final a ser publicada. L. F. Schluckebier participou da concepção e desenvolvimento do modelo analítico de decisão, coleta dos dados de custo, redação e revisão crítica do artigo e aprovação da versão final a ser publicada. R. M. da Silva participou da revisão da literatura, coleta dos dados epimemiológicos, clínicos e da revisão sistemática da acurácia das tecnologias, redação e revisão crítica do artigo e aprovação da versão final a ser publicada. J. U. Braga participou da revisão sistemática da acurácia das tecnologias, redação e revisão crítica do artigo e aprovação da versão final a ser publicada. C. R. G. Bastos participou da revisão da literatura, concepção e desenvolvimento do modelo analítico de decisão, coleta dos dados clínicos e revisão sistemática da acurácia das tecnologias, redação e revisão crítica do artigo e aprovação da versão final a ser publicada. 


\section{Cost-effectiveness of the use of positron emission tomography in the detection of recurrence of differentiated thyroid cancer}

The positron emission tomography $\left({ }^{18} \mathrm{FDG}-\mathrm{PET} / \mathrm{CT}\right)$ was recently introduced in Brazilian health care for many oncology indications but accompanied by higher costs. In our study we performed a cost-effectiveness analysis of the addition of PET/CT to the conventional diagnostic work-up to detect recurrent differentiated thyroid cancers. The analytical decision model represented a hypothetical cohort of adults, thyroid cancer patients with high risk by initial stratification, submitted to total thyroidectomy and ablation with I ${ }^{131}$. The addition of PET/CT was applied to subjects with negative results on $\mathrm{I}^{131}$ scintigraphy. The model was designed from the perspective of the Brazilian public health care system, with a time horizon of 10 years. Effectiveness was measured by the additional recurrent cases detected. Only direct medical costs were considered. Costs and benefits were discounted by $5 \%$. Univariate deterministic and probabilistic sensitivity analyzes were performed to explore the uncertainties. The PET/CT diagnosed 13 additional cases compared to conventional strategy $(1,888$ vs 1,875$)$ by a cost of $\mathrm{R} \$ 477,633.05$ per case detected. The parameters of greatest impact in the sensitivity analysis were the accuracy of conventional tests, cost of PET/CT and the discount rate. The costs of adding PET/CT seems significant and its introduction is not cost-effective on the Brazilian perspective.

> Key words: thyroid cancer; differentiated thyroid carcinoma; cost-effectiveness analysis; ${ }^{18}$ F-FDG-PET; positron emission tomography. 